ASM Sc. J., 13, 2020

https://doi.org/10.32802/asmscj.2020.sm26(2.14)

\title{
Making Sense of Mathematics through Perception, Operation \& Reason: The Case of Divisibility of a Segment
}

\author{
Ng Lee Fong ${ }^{1 *}$, Vincent Pang² and Chin Kin Eng3 \\ ${ }^{1}$ Research and Innovation Department, Institute of Teacher Education Kent Campus, \\ 89207 Tuaran, Sabah, Malaysia \\ ${ }^{2}$ Centre for Teaching Excellence and Academic Quality, Universiti Malaysia Sabah, \\ 88400 Kota Kinabalu, Sabah, Malaysia \\ ${ }^{3}$ College of Education, Psychology \& Social Work, Flinders University, \\ Sturt Rd, Bedford Park SA 5042, Australia
}

\begin{abstract}
The conception of infinity as a process (potential infinity) or as an object (actual infinity) is important for students to acquire understanding in many other related areas in mathematics. This study attempts to describe the infinite divisibility thinking of mathematics student teachers in an Institute of Teacher Education in Malaysia by making sense of mathematics through perception, operation and reason. Data were collected through a self-reporting questionnaire that was administered to 238 elementary school pre-service teachers from selected Teacher Education Institutes in Malaysia. Researchers categorised qualitatively different types of thinking and reported them by using descriptive statistics. The result revealed that the percentage of respondents who conceived infinity as an object was just slightly lower as compare to the percentage of respondents who conceived infinity as a process. Additionally, this study found that there were respondents with problematic conceptions as shown by their inconsistent answers. The open-ended explanations given by all the respondents revealed that most of the pre-service teachers used perception to make meaning on finite and infinite divisibility.
\end{abstract}

Keywords: infinity, teachers' perceptions, problematic conception, actual and potential Infinity

\section{INTRODUCTION}

Calculus involves the concepts of limit, infinity, and real numbers. According to Monaghan (1986), many students experienced difficulty with the algebraic manipulation involved in the first calculus course due to cognitive difficulties with the concepts of limits, infinity and in real numbers. On the other hand, little attention is paid to the notion of infinity in the school mathematics. Certainly, theorems and proofs are taught besides solving procedures, but the coherent and harmonic structure has been diluted from the system (Fischbein et. al., 1995). This study will use the term of perception, operation, and reason as the main theoretical framework to explore how the participants make sense of infinity. In this paper, we will report the data collected from an item of the questionnaire which is related to the infinite divisibility of a segment. The collected student teachers' responses were split into two main opposite categories; those accepting the infinite divisibility and those rejecting it. The infinity conceptions are categorised as potential infinity or as actual infinity. In fact, the construct of divisibility is only one of the ten constructs of the research instrument. The divisibility construct is chosen because it allows participants to embody their infinity thinking through senses, actions and reasoning.

\section{RESEARCH OBJECTIVE}

This study attempts to describe the infinite divisibility thinking (types of infinity thinking) of mathematics student

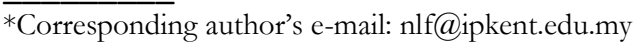


teachers in an Institute of Teacher Education in Malaysia based on the three ways of sense making as proposed by Chin and Tall (2012) and Chin (2013) namely perception, operation and reason. Hence, the theoretical framework in this study was adopted from Chin and Tall (2012) and Chin (2013).

\section{LITERATURE REVIEW}

Chin and Tall (2012) suggested that there were three ways for humans to make sense of mathematics. They claimed that new meanings in new situations may be realized through perception by doing physical manipulation or thought experiment. The first way of making sense is through perception which is essentially about drawing conclusion through senses. This implies that humans make meaning based on what they perceive and experience both in the physical world and the mental world. This is the basic way of thinking. The embodied world includes mental perception (relating to the real-world objects) and the internal conceptions (including visuospatial imaginary) (Tall, 2004).

The second way of sense making is called operation. It is a physical action such as measuring, counting, and symbolising things as a manipulable mental concept in the form of operational symbolism (arithmetic or algebra). Thus, Chin and Tall (2012) described compression of knowledge as a phenomenon when some kinds of symbols are used in the mind to represent actions and concepts. The brain will make sense of the information received and give a name to the concept. Then this concept can be acted upon and can be discussed about its properties.

The third world is related to building formal knowledge in axiomatic systems based on formal definitions and proof (Tall, 2007). Reasoning in school grows in many ways, including making practical definitions and principles as well as using the principles and formulas to determine the rules properly. There are blends of human embodiment, operational symbolism, and axiomatic formalism in making sense of mathematics (Tall, 2013). The supportive conceptions support future learning and problematic conceptions impede sense making. In this case, supportive conceptions refer to conceptions that work in an old context and continue to work in a new context (Chin, 2013). As a consequence of this, problematic conceptions will cause confusion and anxiety in mathematics (Chin \& Tall, 2012; Tall et. al., 2014). These revealed that there must be at an older age to understand the concept of infinity. Reasoning normatively about infinite iterative processes can help students in developing normative conceptions of all aspects of infinity and the mathematical concepts related to it such as infinite sequences and their limits (Lakoff \& Núñez, 2000).

There are two basic concepts of infinity; actual and potential infinity (Fischbein, 2002; Kattou et. al., 2010). In this respect, potential infinity refers to an on-going activity that never ends, whereas actual infinity represents the definite entity which encompasses with potential (Dubinsky et. al., 2005). Furthermore, Fischbein (2002, p309) identified that "actual infinity as what our intelligence finds difficult, even impossible to grasp; the infinity of the world, the infinity of the number of points in a segment, the infinity of real numbers as existing”. It took so many centuries for the notion of actual infinity to be accepted as being possible for both mathematically and cognitively (Dubinsky et. al., 2005).

On the other hand, the concept of potential infinity deals with a dynamic form of infinity. The processes of the situation are the focus, which is at every moment, they are known as an object, but continue endlessly with a new object. When a learner is able to conceive infinity as an object, the potentially infinite process may somehow turn into actual infinity. An example of actual infinity is a set of natural numbers. When we continuously count more and more elements of the set, we need to conceptualise the process as if it will finish somewhere in the set (Lakoff \& Núñez, 2000).

\section{METHODOLOGY}

A. Sample

The 238 samples were taken by purposive sampling from semester two to semester seven majoring mathematics student teachers from 13 campus of Teacher Education Institute in Malaysia. The respondents were in the age range of 20 to 24 years old. They are had taking calculus course and still studying undergraduate mathematics courses in Teacher Education Institute.

\section{B. Instrument}

The instrument was an open-ended questionnaire of three items as shown in Table 1. The items examined the student teachers' conceptions about the infinite divisibility of a segment. Analysis of the responses is reported in order to demonstrate how these respondents perceive and make 
sense particularly in infinity. The open-ended answers were coded into pattern and named according to the theme of sense making (perception, operation and reason)

\section{FINDINGS AND DISCUSSION}

Table 2 shows the explanations offered by the respondents who were categorised into three types of sense making in mathematics through perception, operation and reason. Table 3 shows the results of the dichotomous answer given by the respondents and Table 4 shows the three types of infinity thinking as the result the data analysis.

The open data analysis was difficult to perform because the researchers faced interpretive dilemmas particularly in deciding how a respondent made sense of a particular mathematics question. Making sense through perception, operation and reason are not totally distinct from each other thus the researchers only managed to categorise the responses based on the key words in these responses. In order to solve this issue, the researchers triangulated their analysis with each other then they discussed and arrived at a consensus for those cases in dilemma. On top of that, by using deep reflections method, researchers solved the dilemma (Ng et. al., 2016).

Result in Table 2 shows that for item 1a, 34.03\% of the respondents used their perception to make sense that the process of dividing a segment. On the other hand, for those who think that the process of dividing will be continuously without ending, $15.13 \%$ respondents made sense through reason. Two typical responses are "numbers are unlimited, and the fragments will continuously divide even though the process cannot see by eyes". There were $13.02 \%$ of the respondents made sense through operation claimed that the division is always infinity.

The result for item $1 \mathrm{~b}$ is almost similar to item $1 \mathrm{a}$. Justifications provided for the responses were also similar to those of the previous item and the highest percentage is making sense through perception. For item 1c, majority of the respondents used perception to make sense of their response. When respondents define the divisibility of a segment is having an end (an actual infinity), they used phrases such as "the segment is limited with no more space between two points", "the division will become unlimited and unable to divide anymore"to explain their answer. On the other hand, if the respondents conceived the division of a segment as a continuous process, their phrases were "the number of division is infinite" and "fragments are continuously dividing even though the process cannot be seen with the eyes".

Based on the findings in Table 2, for those who had actual infinity thinking, most of them made sense through perception. The student teachers in this study agreed that the process of the division will not be able to divide further and come to an end. Additionally, they also explained their responses based on their knowledge and experience with infinity (Ng et. al., 2015). These respondents, based on their perception, explained using simple sentences without support from any calculations or definitions. However, the findings reveal that for those who think that the process of dividing will be continuous, the best way of making sense is through operation followed by reason. These groups of respondents were mainly able to see the division is in symbolism of number with process and they stated as "the numbers of the division is infinity".

Table 3 shows that $46.64 \%$ respondents agreed that the process of diving will come to an end while $53.36 \%$ respondents professed that the dividing process will go on continuously for item $1 \mathrm{a}$. The responses of Item $1 \mathrm{~b}$ were almost similar to the responses of item 1a denoted by $42.86 \%$ and $57.14 \%$. Item $1 \mathrm{c}$ asked the respondents to compare the divisibility of a segment by using two parts and three parts. In this respect, there was an increase in the number of respondents who agreed (answered "yes") to the statement of Item $1 \mathrm{c}$ with $55.04 \%$ respondents believed that the process of division by three will end faster as compared to continuously divided by two and they possessed actual infinity.

The thinking behind the collected responses was categorized into three types based on the agreement given by the respondents for statements of Item 1a, $1 \mathrm{~b}$ and 1c. Based on Table 4, it can be concluded that $38.2 \%$ individuals agreed (answered "yes") to all the statements given, this category of respondents were claimed to have actual infinity thinking. In contrast, 40.8\% respondents disagreed with all the statements given. In this case, they responded "no" for all the statements. This group of respondents was categorized as having potential infinity thinking.

In addition, there were $21.0 \%$ responded inconsistently as they agreed to some statements and disagreed to some statements at the same time. In this study, problematic conceptions refer to conceptions with hidden cognitive conflicts that lead to problematic thinking. Inconsistent responses are a strong indicator for the existence of problematic conceptions. This study has identified that there 
were $21.0 \%$ respondents with problematic thinking based on the collected responses.

The result in Table 4 shows that there were more respondents, who held potential infinity thinking as compared to those who held actual infinity thinking. This finding was congruent to other findings (Kattou et. al., 2010; Monaghan, 2001). From the view of the lens of English adjective, infinite means endless or having no end and in English noun, infinity means the property of having no end (Baber, 2011). Therefore, more people will conceive infinity in potential thinking. The English word "infinity" influences the learners' conception of infinity (Baber, 2011). The definition given by some dictionaries is confusing the readers when infinity is defined as indefinitely large number or amount. Such definition is misleading and mathematically wrong because it is meaningless as no number and no amount can be defined as indefinitely large (Baber, 2011).

According to Fishbein (2002), the humans' mind finds it difficult to distinguish between actual and potential infinity. He believed that our intelligence is essentially adapted to finite realities because our logic concerns about laws that are able to handle only concepts expressed by finite realities. Therefore, it is harder to accept actual infinity as compared to potential infinity. He explains that in potential infinity we cannot visualised, but we are able to conceive the idea of after every natural number, no matter how big the number, there is another natural number. Tall (2008) believes there is a transition from one concept to another concept in developing formal mathematical thinking. Thus, the routine actions are important as the concept images are formed by individuals' mental structure. For instance, when an individual thinks that each counting number is followed by one after another, this situation leads to potential thinking. This is the same as when student teachers conceived that the divisibility of a segment will always be able to be divided repeatedly. If individuals are thinking in terms of categorising a collection of numbers and giving it a name or a symbol, this shows that they have actual infinity. In this study, the notion of actual infinity thinking emerges when the student teachers conceive that the segment will stop dividing at a specific point, as they may think that it cannot be divided further and this gives rise to actual infinity.

Based on the result, a new category is created and named as problematic thinking to represent a group of respondents who have given inconsistent answers denoted by $21.0 \%$ respondents. These results revealed that some student teachers changed their answers from potential infinity to actual infinity or vice versa across the three items in Question 1, and this indicated the instability of thinking of the respondents.

\section{CONCLUSION}

Humans make sense of infinity through perception, operation and reason regardless of the kinds of infinity thinking that they have. The data indicated that student teachers mostly made sense through perceptions to explain their answers rather than through operation and reason. Based on the divisibility of a segment item, three types of infinity thinking were identified: potential thinking, actual thinking and problematic thinking. Teachers' guidance to formal education knowledge is essential. How teachers teach a concept is important. This situation shows that in order to shift from one context to another context, it would lead to supportive conception or problematic conceptions in future. The important aspects in mathematical thinking are related to the ways that students make sense of mathematics (Chin \& Tall, 2012). On top of that, teachers need be aware of the needs of the students, encouraging them to cope with the changes of meanings across different contexts.

The notion of problematic thinking in this paper reveals our student teachers' view of divisibility of infinity was inconsistently and they are mixed thinking between actual and potential. The finding shows that student teachers' sense making need to be improve. Therefore, sense making of mathematics affecting the supportive and problematic conceptions and advanced ideas in the conception in certain topics will affect the sense making in a new topic or new context (Chin, 2014). The institution of teacher education in Malaysia should come out with proactive courses that enhanced the sense making not only in mathematics program but also in another subject program.

\section{ACKNOWLEDGEMENT}

The authors would like to express sincere gratitude to the Malaysia Ministry of Education, Institute of Teacher Education Malaysia and Universiti Malaysia Sabah for providing the financial means. 


\section{REFERENCES}

Baber, R. L. 2011, The language of mathematics: Utilizing Math in practice. Hoboken, A John Wiley \& Son, New York.

Chin, K. E. 2013, Making sense of mathematics: supportive and problematic conceptions with special reference to trigonometry. Unpublished PhD Thesis, Warwick Institute of Education: University of Warwick.

Chin, K. E. 2014, Supportive and problematic aspects in mathematical thinking over the longer term. In Oesterle, S., Nicol, C., Liljedahl, P., \&Allan, D. (Eds.). Proceedings of the 38th Conference of the International Group for the Psychology of Mathematics Education, 6, 41. Canada.

Chin, K. E. and Tall, D. 2012, Making sense of mathematics through perception, operation \& reason: The case of trigonometric functions. In Tai-Yih Tso (Ed.). Proceedings of the 36th Conference of the International Group for the Psychology of Mathematics Education, 4, 264, Taiwan.

Dubinsky, E., Weller, K., McDonald, M. A., and Brown, A. 2005, Some historical issues and paradoxes regarding the concept of infinity: An Apos-Based Analysis: Part 1. Educational Studies in Mathematics,58 (3), 335-359.

Fischbein, E. 2002, Tacit model and infinity. Kluwer Academic Publishers, Netherlands.

Fischbein, E., Jehiam, R. and Cohen, D. (1995). Concept of Irrational Numbers in High-School Students and Prospective Teachers. Educational Studies in Mathematics,29 (1), 29-44.

Kattou, M., Michael, T., Kontoyianni, K., Christou, C. and Philippou, G. 2010 Teachers' perceptions about infinity: A process or an object? Proceedings of the Sixth Congress of the European Society for Research in Mathematics Education (Proceedings of CERME 6). January 28thFebruary $1^{\text {st }}$ 2009. Lyon France: 1771-1780. www.inrp.fr/editions/cerme6.

Lakoff, G. and Nunez, R. 2000, Where mathematics come from. Basic Books, New York.

Monaghan, J. 1986, Adolescents' understanding of limits and infinity. Unpublished PhD Thesis, University of Warwick. United Kingdom.

Monaghan, J. 2001, Young peoples' ideas of infinity. Educational Studies in Mathematics, 48, 239-257.

Ng, L. F., Chin, K. E. and Pang, V. 2016, Making sense of infinity through perception, operation and reason. Man In India, 96 (1-2), 447-455.

Ng, L. F., Pang, V. and Chin, K. E. 2015, Is infinity an object or process? Proceeding of $1^{\text {st }}$ Borneo International Conference on Science and Mathematics Education. 21-13 September 2015. University Malaysia Sabah: Malaysia.

Tall, D. 2004, Introducing three worlds of mathematics. For the Learning of Mathematics, 23 (3), 29-33.

Tall, D. 2007, Developing a theory of Mathematical growth. ZDM -The International Journal of Mathematics Education,39(1-2), 145-154.

Tall, D. 2008, The transition to formal thinking in mathematics. Mathematics Education Research Journal, 20 (2), 5-24.

Tall, D. 2013, Making sense of Mathematical reasoning and proof. In Michael N. Fried \& Tommy Dreyfus (Eds.). Mathematics \& Mathematics Education: Searching for Common Ground: 1-13. Springer, New York.

Tall, D., Lima, R. N. and Healy, L. 2014, Evolving a threeworld framework for solving algebraic equations in the light of what a student has met before. Journal of Mathematical Behavior, 34, 1-13. 


\section{Appendix: Tables}

Table 1: The Question 1 and sub-items

Question 1 A segment $\mathrm{AB}$ is divided into two equal parts (Figure 1). Point $\mathrm{M}$ is the midpoint of the segment. Now
we divide $\mathrm{AM}$ and $\mathrm{MB}$. Point $\mathrm{F}$ and $\mathrm{G}$ represent the midpoints of the segments $\mathrm{AM}$ and $\mathrm{MB}$,
respectively. The processes of dividing continuously go on. With each division, the fragments become
smaller and smaller.
Item 1a
Will we arrive at a situation such that the fragments will be so small that we will not be able to divide
further? Explain your answers
Consider again the segment AB of question 1 (a). This time, divide it into three equal parts. The
processes of dividing continuously go on. With each division, the fragments become smaller and
smaller. Will we arrive at a situation such that the fragments will be so small that we will not be able
to divide further? Explain your answers
Will we arrive at a situation such thatdivide by three equal parts will sooner get smaller until not
able to continue dividing compare to 1 (a)

Table 2: The summary of answers and explanations given by 238 respondents.

\begin{tabular}{cccccc}
\hline Item & Answer & Perception(\%) & Operation(\%) & Reason(\%) & No explanation(\%) \\
\hline 1a & Yes- 46.64 & 34.03 & - & 2.10 & 10.51 \\
& No- 53.36 & 10.50 & 13.02 & 15.13 & 14.71 \\
\multirow{2}{*}{ b } & Yes- 42.86 & 29.00 & - & 1.68 & 12.18 \\
& No- 57.14 & 15.12 & 7.57 & 13.44 & 21.01 \\
\multirow{2}{*}{ 1c } & Yes- 55.04 & 21.01 & 10.5 & 7.98 & 15.55 \\
& No- 44.96 & 16.81 & 6.31 & 2.94 & 18.91 \\
\hline
\end{tabular}

Table 3: The percentage of agreements on dichotomous answer

\begin{tabular}{clc}
\hline Item & Answer of responses & \% \\
\hline 1a & Yes, the process of dividing will come to an end (Actual) & 46.64 \\
& No, the process of dividing will continuously go on (Potential) & 53.36 \\
\multirow{2}{*}{ 1b } & Yes, the process of dividing will come to an end (Actual). & 42.86 \\
& No, the process of dividing will continuously go on (Potential). & 57.14 \\
1c $\quad$ Yes, the process of division by three will end faster compared to the division by two & 55.04 \\
& (Actual). & 44.96 \\
\hline
\end{tabular}


Table 4: Types of infinity thinking

\begin{tabular}{lc}
\hline \multicolumn{1}{c}{ Type of Infinity Thinking } & $\%$ \\
\hline Actual Infinity Thinking & 38.2 \\
Potential Infinity Thinking & 40.8 \\
Problematic Thinking & 21.0 \\
\hline Total & 100.0 \\
\hline
\end{tabular}

